\title{
RESEARCH
}

\section{Photodynamic therapy enables tumor-specific ablation in preclinical models of thyroid cancer}

\author{
Nidal Muhanna1,2,3,*, Harley H L Chan 1,2,*, Jason L Townson ${ }^{1,2}$, Cheng S Jin 1,2, Lili Ding', Michael S Valic', \\ Catriona M Douglas ${ }^{1,2}$, Christina M MacLaughlin1, Juan Chen ${ }^{1}$, Gang Zheng1,4 and Jonathan C Irish ${ }^{1,2}$ \\ ${ }^{1}$ Guided Therapeutic (GTx) Program, TECHNA Institute, Princess Margaret Cancer Center and University Health Network, Toronto, Ontario, Canada \\ 2Department of Otolaryngology-Head and Neck Surgery-Surgical Oncology, Princess Margaret Cancer Centre/University Health Network, University of \\ Toronto, Toronto, Ontario, Canada \\ ${ }^{3}$ Department of Otolaryngology-Head and Neck Surgery, Tel Aviv Sourasky Medical Center, Tel Aviv University, Tel Aviv, Israel \\ 4Department of Medical Biophysics, University of Toronto, Toronto, Ontario, Canada
}

Correspondence should be addressed to J Chen or G Zheng or J C Irish: juan.chen@uhnresearch.ca or gang.zheng@uhnres.utoronto.ca or Jonathan.Irish@uhn.ca

*(N Muhanna and $\mathrm{H}$ Chan contributed equally to this work)

\begin{abstract}
The incidence of differentiated thyroid cancer has increased significantly during the last several decades. Surgical resection is the primary treatment for thyroid cancer and is highly effective, resulting in 5-year survival rates greater than $98 \%$. However, surgical resection can result in short- and long-term treatment-related morbidities. Additionally, as this malignancy often affects women less than 40 years of age, there is interest in more conservative treatment approaches and, an unmet need for therapeutic options that minimize the risk of surgery-related morbidities while simultaneously providing an effective cancer treatment. Photodynamic therapy (PDT) has the potential to reduce treatment-related side effects by decreasing invasiveness and limiting toxicity. Owing to multiple advantageous properties of the porphyrin-HDL nanoparticle (PLP) as a PDT agent, including preferential accumulation in tumor, biodegradability and unprecedented photosensitizer packing, we evaluate PLP-mediated PDT as a minimally invasive, tumorspecific treatment for thyroid cancer. On both a biologically relevant human papillary thyroid cancer (K1) mouse model and an anatomically relevant rabbit squamous carcinoma (VX2)-implanted rabbit thyroid model, the intrinsic fluorescence of PLP enabled tracking of tumor preferential accumulation and guided PDT. This resulted in significant and specific apoptosis in tumor tissue, but not surrounding normal tissues including trachea and recurrent laryngeal nerve (RLN). A long-term survival study further demonstrated that PLP-PDT enabled complete ablation of tumor tissue while sparing both the normal thyroid tissue and RLN from damage, thus providing a safe, minimally invasive, and effective alternative to thyroidectomy for thyroid cancer therapies.
\end{abstract}

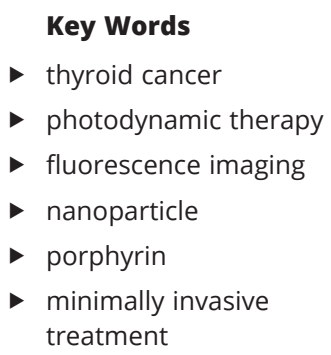

Endocrine-Related Cancer (2020) 27, 41-53

\section{Introduction}

Thyroid cancer is the most prevalent endocrine cancer worldwide, with the incidence more than doubling in many countries since the early 1990s (La Vecchia et al.
2015, Lim et al. 2017). Among various subtypes of thyroid cancer (papillary, follicular, medullary and anaplastic), papillary thyroid cancer (PTC) is the most prevalent, c) 2020 Society for Endocrinology Published by Bioscientifica Ltd. Printed in Great Britain 
accounting for $80-85 \%$ of thyroid cancers. The increasing frequency of PTC, due in part to improved detection of small nodules, is partially responsible for the rise in incidence of thyroid cancer worldwide (La Vecchia et al. 2015). Despite the increasing incidence of PTC, the long-term survival is excellent, with 5-year survival rates reported as 98\% (La Vecchia et al. 2015).

Surgical resection of the thyroid is the current gold standard PTC treatment, with or without thyroid remnant ablation with radioactive iodine and thyroid hormone suppression therapy (Haugen et al. 2016). While these treatment approaches are very effective in the vast majority of patients with differentiated thyroid cancer, complications can result from surgical intervention including recurrent laryngeal nerve (RLN) injury, hypoparathyroidism and surgical hemorrhage/ hematoma, occurring in $12.3 \%$ of patients (Papaleontiou et al. 2017). In addition, the surgical scar in the middle of the anterior neck is very prominent cosmetically and can negatively impact the quality of life of patients, many of whom are young female patients. As such, there has been increasing interest in non-invasive treatment approaches. This has led to new surgical techniques, such as transoral and transaxillary thyroidectomy, to prevent visible scarring on the neck. As the overall survival of PTC is very high and disease recurrence low (up to $7 \%$ of patients) following initial surgery (Ito et al. 2012), improvements in treatment methods have increasingly focused on reducing morbidity from treatment, reducing procedure invasiveness and, improving cost in comparison to current standards of treatment while maintaining excellent survival rates. Percutaneous ethanol injection and radiofrequency or laser ablation have been proposed as alternatives (Haugen et al. 2016); however, they are not without morbidity including pain, skin burn and voice changes. This is felt to be due to the lack of specificity for the tumor in comparison to normal thyroid tissue (Pacella et al. 2015). New and innovative treatment alternatives therefore must be sought (Gambelunghe et al. 2013, Pacella et al. 2015).

Photodynamic therapy (PDT) involves the administration of photosensitizers, which when activated with near-infrared laser light produce localized cytotoxic reactive oxygen species (ROS). By combining tumorspecific accumulation of molecular sensitizers and minimally invasive tumor-focused light delivery, PDT can exert tumoricidal effects using lower laser powers while sparing neurological structures and normal tissues (Betz et al. 2007). Therefore, PDT has potential to satisfy the criteria required for improving PTC treatment, with a number of advantages in comparison to surgical intervention and alcohol, radiofrequency or laser ablation. Recently, we have described development and application of a multifunctional porphyrin nanoparticle (PLP) capable of image-guided PDT for treatment of diverse cancers, including head and neck squamous cell cancer (Muhanna et al. 2015a,b). The PLP is a PEG-free, biomimetic, ultrasmall $(<30 \mathrm{~nm})$ nanoparticles composed of densely packed porphyrin phospholipids (Muhanna et al. 2015b). The ultra-small size of PLP (30 nm) enhances the penetration and distribution within the tumor upon accumulation of nanoparticle in the tumor interstitial space. The high-density porphyrin released from PLP is then activated, resulting in potent PDT efficacy to primary tumors and prevention of tumor metastasis without damage of adjacent critical structures (Muhanna et al. $2015 a, b)$. Here we investigate if the PLP-PDT could be a safe and effective therapeutic option to treat differentiated thyroid cancer, while minimizing the risk and morbidities associated with surgical interventions.

\section{Materials and methods}

\section{Synthesis of PLP and ${ }^{64} \mathrm{Cu}$ labeling}

1,2-Dimyristoyl-sn-glycero-3-phosphocholine (DMPC) was purchased from Avanti Polar Lipids Inc. (AL, USA). Pyropheophorbide-lipid (Porphyrin-lipid) was prepared and the protocol was described previously. Cholesteryl oleate was purchased from Sigma-Aldrich Co. The ApoA-1 mimetic peptide (R4F), Ac-FAEKFKEAVKDYFAKFWD, was purchased from GL Biochem Ltd. (Shanghai, China). PLP was prepared following previously reported method (Cui et al. 2015). Briefly, a lipid film was prepared by evaporation of lipid mixtures in chloroform under nitrogen. The lipid mixture for PLP consists of $0.9 \mu \mathrm{mol}$ porphyrin-lipid, $2.1 \mu \mathrm{mol}$ DMPC and $0.3 \mu \mathrm{mol}$ cholesteryl oleate. The completely dried lipid films were hydrated with $1.0 \mathrm{~mL}$ PBS buffer (150 mM, pH 7.5) and sonicated (Bioruptor $\left.{ }^{\circledR}\right)$ at low frequency ( $30 \mathrm{~s} \mathrm{on} / 30 \mathrm{~s}$ off) for 30 cycles at $40^{\circ} \mathrm{C}$. R4F peptide $(2.0 \mathrm{mg}, 5 \mathrm{mg} / \mathrm{mL})$ was titrated into the rehydrated solution. After overnight shaking at $4^{\circ} \mathrm{C}$, the solution was filtered with $0.1 \mu \mathrm{m}$ membrane $\left(\right.$ Millex $^{\circledR}$, Sigma-Aldrich) to produce PLP.

PLP was labeled with ${ }^{64} \mathrm{Cu}$ using the previously reported method (Cui et al. 2015). Briefly, PLP was 1:4 diluted with $0.1 \mathrm{M} \mathrm{NH}_{4} \mathrm{OAC}(\mathrm{pH} 5.5)$, and then mixed with ${ }^{64} \mathrm{CuCl}_{2}$ solution (Mallinckrodt Institute of Radiology, Washington University School of Medicine) and incubated at $40^{\circ} \mathrm{C}$ for 
$60 \mathrm{~min}$. The mixture was purified with the centrifugal units (30K, Amicon Ultra) and the radiochemical purity and yield were assessed.

\section{Cell culture}

The human PTC cell line, K1, was purchased from SigmaAldrich ${ }^{\circledR}$ (92030501-1VL, ECACC origin, STR verified), and cultured in medium of DMEM: Ham's F12: MCDB 105 at a ratio of 2:1:1 with $2 \mathrm{mM}$ Glutamine and $10 \%$ Fetal Bovine Serum (Sigma-Aldrich $\left.{ }^{\circledR}\right)$. MDA-T85 cells (ATCC CRL-3354) were purchased from ATCC and cultured in RMPI-1640 with $2 \mathrm{mM}$ Glutamine and 10\% FBS. All experiment with MDA-T85 were completed within 10 passages and 2 months of arrival from ATCC. STR profiling of K1 was completed by ATCC Cell Line Authentication Services Lab (Manassas, VA, USA), with results attached as Supplementary Fig. 1 (see section on supplementary materials given at the end of this article). The cells were grown in a humidified incubator with $5 \% \mathrm{CO}_{2}$ at $37^{\circ} \mathrm{C}$. To assess K1 and MDA-T85 cell uptake of PLP, cells were incubated with PLP ( $5 \mu \mathrm{M}$ of porphyrin concentration) for $6 \mathrm{~h}$. Cells were then rinsed with PBS twice, re-incubated in fresh medium and imaged by Olympus FV1000 laser confocal scanning microscopy (Olympus) to visualize DAPI (Ex. 405 nm, Em 425-475 nm) and PLP (Ex. 633 nm, Em. 647-747 nm) signals, respectively. To assess K1 and MDA-T85 cell sensitivity to PLP-mediated PDT, cells were incubated for $24 \mathrm{~h}$ with $0.1,0.5,1$ or $5 \mu \mathrm{M}$ PLP or same amount of PBS in standard growth media in a 96-well black-walled plate. Cells were washed three times with PBS and replaced with full fresh medium, and then exposed to $671 \mathrm{~nm}$ light at a dose of $10 \mathrm{~J} / \mathrm{cm}^{2}$ $\left(100 \mathrm{~mW} / \mathrm{cm}^{2}\right)$. Viability was measured at $24 \mathrm{~h}$ post laser treatment using an AlamarBlue assay (Resazurin sodium salt, Sigma-Aldrich).

\section{Animal models}

All animal procedures were performed following protocols approved by University Health Network (UHN) Animal Care Committee.

\section{Mouse model}

The human PTC cell line K1 was used to develop subcutaneous and orthotopic thyroid cancer models in mice. Athymic nude male mice were purchased from Harlan Laboratory and housed in the Animal Research Centre of UHN. For the subcutaneous tumor model, $5 \times 10^{6} \mathrm{~K} 1$ cells were suspended in $100 \mu \mathrm{L}$ PBS and injected to both sides of the mouse flanks subcutaneously, to create a dual-tumor mouse model. The growth of subcutaneous thyroid tumors was then monitored by caliper measurement twice per week, with tumor volume calculated using the formula $\mathrm{L} \times \mathrm{W}^{2} / 2$. For the orthotopic model, $5 \times 10^{5} \mathrm{~K} 1$ cells suspended in $20 \mu \mathrm{L}$ PBS were injected into the left lobe of the thyroid following surgical exposure via incision through skin and salivary glands. Following injection, the incision was sutured and tumor growth was monitored by palpation and MRI (7T, Biospec, Bruker). Mice were injected with PLP when tumors reached 3-7 $\mathrm{mm}$.

\section{Evaluation of therapeutic response of PLP-PDT on a mouse model}

Therapeutic efficacy of PLP-PDT was initially evaluated in mice using the human PTC cell line K1 in a bilateral subcutaneous tumor model. Mice with tumors on both the right and left hind flanks were randomized into two groups when the tumors reached $5 \mathrm{~mm}$ in diameter. One group received $4 \mathrm{mg} / \mathrm{kg}$ PLP via lateral tail vein injection $(n=3)$ while the other group received no injection $(n=3)$. At $24 \mathrm{~h}$ post injection, tumors located on the right flank of all mice were irradiated by laser at 671 $\mathrm{nm}$, for a total light dosage of $100 \mathrm{~J} / \mathrm{cm}^{2}\left(100 \mathrm{~mW} / \mathrm{cm}^{2}\right.$ at the irradiation area of $8 \mathrm{~mm}$ diameter). Tumors on the left flank of these mice were not irradiated and served as a PLP injection control. In the group receiving no PLP, tumor on the right flank was irradiated with the same laser dose as the treatment group (671 nm for the light dosage of $100 \mathrm{~J} / \mathrm{cm}^{2}$ ) and served as laser only control, while the tumor on the left flank served as the untreated control. During each laser treatment, tumor temperature was monitored by a NIR thermal camera (Mikroshot ${ }^{\circledR}$, LUMASENSE Technologies, Santa Clara, CA, USA) once every $4 \mathrm{~min}$. All mice were killed at $24 \mathrm{~h}$ post treatment, and tumors dissected and fixed in $10 \%$ formalin for histology analysis, including H\&E and TUNEL.

\section{Rabbit model (VX2)}

The rabbit VX2 tumour model, derived from a papillomavirus-associated rabbit squamous cell carcinoma, has been used for decades as a pre-clinical model of multiple types of cancer, including liver, kidney, brain, pancreas and head and neck cancer (Rous et al. 1952, Lin et al. 2009, Parvinian et al. 2014). While not a papillary thyroid carcinoma, growth of 
VX2 tumours in rabbit thyroid provides anatomically relevant tumors and normal tissues of sufficient size to allow assessment of PDT on both normal tissue (thyroid, trachea, RLN and vocal cords) and VX2 tumor. For the purpose of thyroid ablation, the VX2 model allowed assessment of longitudinal survival in a more scale-appropriate animal model. To develop a rabbit (anatomically relevant) VX2 thyroid tumor model, following pre-operative antibiotic and analgesic administration, the neck area of 2.5-3 kg New Zealand white rabbits (Charles River, Wilmington, MA, USA) was shaved and prepared for sterile surgery. VX2 tumor cells were prepared from filtered homogenate of frozen VX2 tumors resuspended in HBSS. Cells were injected into the thyroid by making an incision of $2 \mathrm{~cm}$ in length through the skin and underlying strap muscle in order to expose the right lobe of thyroid. $100 \mu \mathrm{L}$ of VX2 cell suspension $\left(\sim 2 \times 10^{6}\right.$ cells $)$ was then injected into the right lobe of the thyroid gland. Immediately following injection, the neck muscle and skin incisions were closed with sutures and the animals were recovered with $100 \%$ oxygen for at least $30 \mathrm{~min}$. Rabbits were treated with oral analgesic postoperatively for 3 days while recovering from the surgery, and skin stitches were removed 10 days following surgery. The growth of the anatomically relevant VX2 thyroid cancer was monitored using CT twice a week after the surgery.

\section{CT imaging for monitoring rabbit anatomically relevant thyroid tumor and lymph node}

All tumor-bearing rabbits were imaged by MicroCT (Locus Ultra, GE Medical Systems) to longitudinally monitor the size of thyroid tumors and cervical lymph nodes. Under isoflurane anesthesia, rabbits were placed in a supine position on the scanning bed of the MicroCT. Immediately prior to image acquisition, animals were intravenously injected with $10 \mathrm{~mL}$ Omnipaque (GE Healthcare) at $350 \mathrm{mg} / \mathrm{mL}$ over $1 \mathrm{~min}$ following $2 \mathrm{~mL}$ $0.2 \%(2 \mathrm{mg} / \mathrm{mL})$ heparin sodium salt. MicroCT scans were acquired at $80 \mathrm{kvp}$ and $50 \mathrm{~mA}$, images were reconstructed using traditional filter back projection with graphic processing unit (GPU) improving image reconstruction speed, image resolution $0.154 \times 0.154 \mathrm{~mm}$ pixel size and slice thickness in $0.154 \mathrm{~mm}$. Afterward, all CT-based images were analyzed using Microview (GE Healthcare) and a custom in-house program written using MATLAB (MathWorks®, Natick, MA, USA). The mean and standard deviation of the voxel signal distribution within each volume of interest (VOI) was calculated.

\section{Fluorescence imaging of rabbit thyroid tumor}

When tumors reached $1 \mathrm{~cm}$ diameter at 1-2 weeks after tumor inoculation, PLP was injected intravenously through a catheter in rabbit marginal ear vein at the dose of $4 \mathrm{mg} / \mathrm{kg}$. At $24 \mathrm{~h}$ post administration of PLP, rabbits were anesthetized and an incision of $2 \mathrm{~cm}$ length was made on the neck area of the rabbit, and an in-house fluorescence imaging endoscopy system $(650 \pm 20 \mathrm{~nm}$ excitation, $700 \pm 25 \mathrm{~nm}$ emission) was used to image the area of interest to detect the thyroid tumor. The fluorescent area was dissected and then imaged by Maestro ${ }^{\circledR}$ (Caliper Life Sciences). To confirm the accumulation of PLP in the thyroid tumor, rabbits were killed for ex vivo fluorescence imaging, and the dissected tissues were frozen in OCT gel in liquid nitrogen. Frozen samples were then cut and stained with hematoxylin and eosin (H\&E), pancytokeratin (AE1/AE3) and DAPI using the adjacent slides of $6 \mu \mathrm{m}$ thickness.

\section{Evaluation of PLP-PDT therapeutic efficacy by long-term survival study on rabbit VX2 thyroid tumor}

For survival studies, rabbits were randomly assigned to one of three longitudinal treatment groups when tumors reached an approximate $1 \mathrm{~cm}$ diameter: an untreated control group; a laser-only treatment group, and a PLPPDT treatment group. To treat VX2 thyroid tumors, rabbits were anesthetized and the thyroid bed was exposed via a $2 \mathrm{~cm}$ incision through neck skin and strap muscle. Prior to initiation of laser exposure and PDT, in vivo fluorescence imaging was conducted using a NOVADAQ Pinpoint system (Mississauga, Ontario) to visualize PLP fluorescence and thyroid tumor co-localization in the PLP-PDT group. For the PDT group, treatment was completed using laser treatment with the guidance of fluorescence via laser fiber insertion in the tumor for intra-tumor irradiation at a light dose of $100 \mathrm{~J} / \mathrm{cm}$ and laser power of $100 \mathrm{~mW} / \mathrm{cm}$. During the laser irradiation, temperature changes of tumors were monitored using a near infrared thermal camera, and the temperature increase of laser only treatment and PLPPDT was then quantitatively compared. Tumors of these rabbits were imaged by CT twice a week, and tumor size was quantified as described earlier. The endpoint of the survival study was defined when tumor size reached $3 \mathrm{~cm}$ diameter or any abnormal physiological or behavioral sign was observed, such as the decrease of body weight or difficulty eating after the treatment. Endoscopy was performed using standard methods of fiber optic laryngoscopy to visualize vocal cord movements. 


\section{Statistical analysis}

Statistical analysis was completed using a two-tailed $t$-test and GraphPad Prism or Microsoft Excel software. $P$ values less than 0.05 were considered significant and error bars represent standard deviations.

\section{Results}

Evaluation of the PLP fluorescence-guided PDT treatment was completed using PTC cell lines in vitro, and both mouse (biologically relevant human PTC) and rabbit (anatomically-relevant VX2 tumor-implanted in thyroid) models of thyroid cancer. PLP-PDT-mediated treatment response and cell toxicity were assessed by post-treatment histology analysis of tumor tissue, the recurrent laryngeal nerve (RLN), trachea and thyroid gland. Endoscopic assessment of vocal cord mobility was used to assess nerve function. The results demonstrate a highly selective, safe and efficient treatment of thyroid tumors by PLP-enabled fluorescence-guided PDT.

\section{In vitro results}

\section{Human papillary thyroid carcinoma cell line PLP uptake and PDT sensitivity in vitro}

The efficiency of K1 and MDA-T85 cellular uptake of PLP was evaluated in vitro by co-incubation of particles and cells for $6 \mathrm{~h}$, with cells subsequently exhibiting significant intracellular porphyrin fluorescence (Fig. $1 \mathrm{~A}$ and B). Exposure to 671 laser light at $10 \mathrm{~J} / \mathrm{cm}^{2}$ was found to result in significant $(P<0.05$ and 0.01 as indicated) loss of cell viability in $\mathrm{K} 1$ and MDA-T85 cells at $24 \mathrm{~h}$ post treatment, but not cells incubated with PLP without exposing to laser light (Fig. 1C and D).

\section{Mouse model results}

\section{PLP-enabled fluorescence image-guided PDT and ${ }^{64} \mathrm{Cu}$ PLP biodistribution in biologically relevant mouse models of thyroid cancer}

Accumulation of PLP in tumor and PLP-mediated PDT were first evaluated in a subcutaneous $\mathrm{K} 1$ papillary thyroid cancer mouse model when tumors reached $5 \mathrm{~mm}$ in diameter. The accumulation of PLP in tumors following systemic administration ( $4 \mathrm{mg} / \mathrm{kg}$ PLP) was monitored by PLP-enabled fluorescence imaging. At $24 \mathrm{~h}$ post injection, porphyrin fluorescence signal was detected from the subcutaneous tumor while the surrounding tissue exhibited minimal fluorescence (Fig. 2A), indicating tumor preferential accumulation of PLP in the subcutaneous PTC tumor. PDT was initiated by irradiating tumors using a 671 $\mathrm{nm}$ laser at $100 \mathrm{~mW} / \mathrm{cm}^{2}$ with a light dose of $100 \mathrm{~J} / \mathrm{cm}^{2}$. Mice bearing bilateral hind flank tumors were randomly assigned one of four treatment groups: no-treatment control (no-PLP, no laser), laser control (no PLP, laser treatment), PLP control (with PLP, no laser irradiation), and PLP-PDT (with PLP and laser treatment). During laser irradiation,

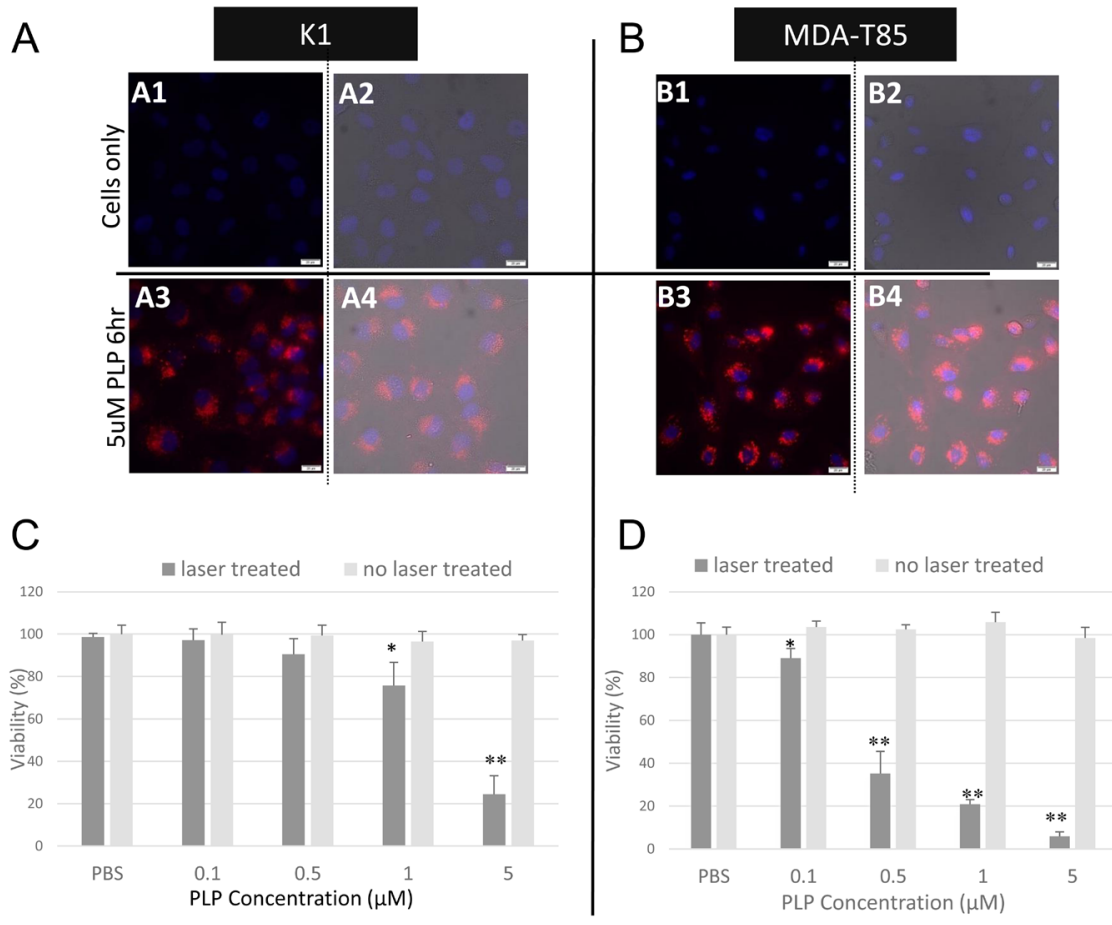

Figure 1

PLP internalized in a human PTC cell lines and induced effective PDT response. (A and $B$ ) Representative fluorescence micrographs of K1 and MDA-T85 cell uptake of PLP by 6 -h incubation, indicating the efficiency of K1 and MDA-T85 cellular uptake of PLP in vitro (porphyrin fluorescence signal: red; DAPI signal: blue). (C and D) The PLP-PDT efficacy on K1 and MDA-T85 cells was investigated by measuring the cell viability with and without laser treatment using MTT assay. PDT treatment method: $671 \mathrm{~nm}$ laser at 10 $\mathrm{J} / \mathrm{cm}^{2}$. Cell viability was normalized to that of the untreated cells in medium ( $* P<0.05 ; * * P<0.01$ ). 


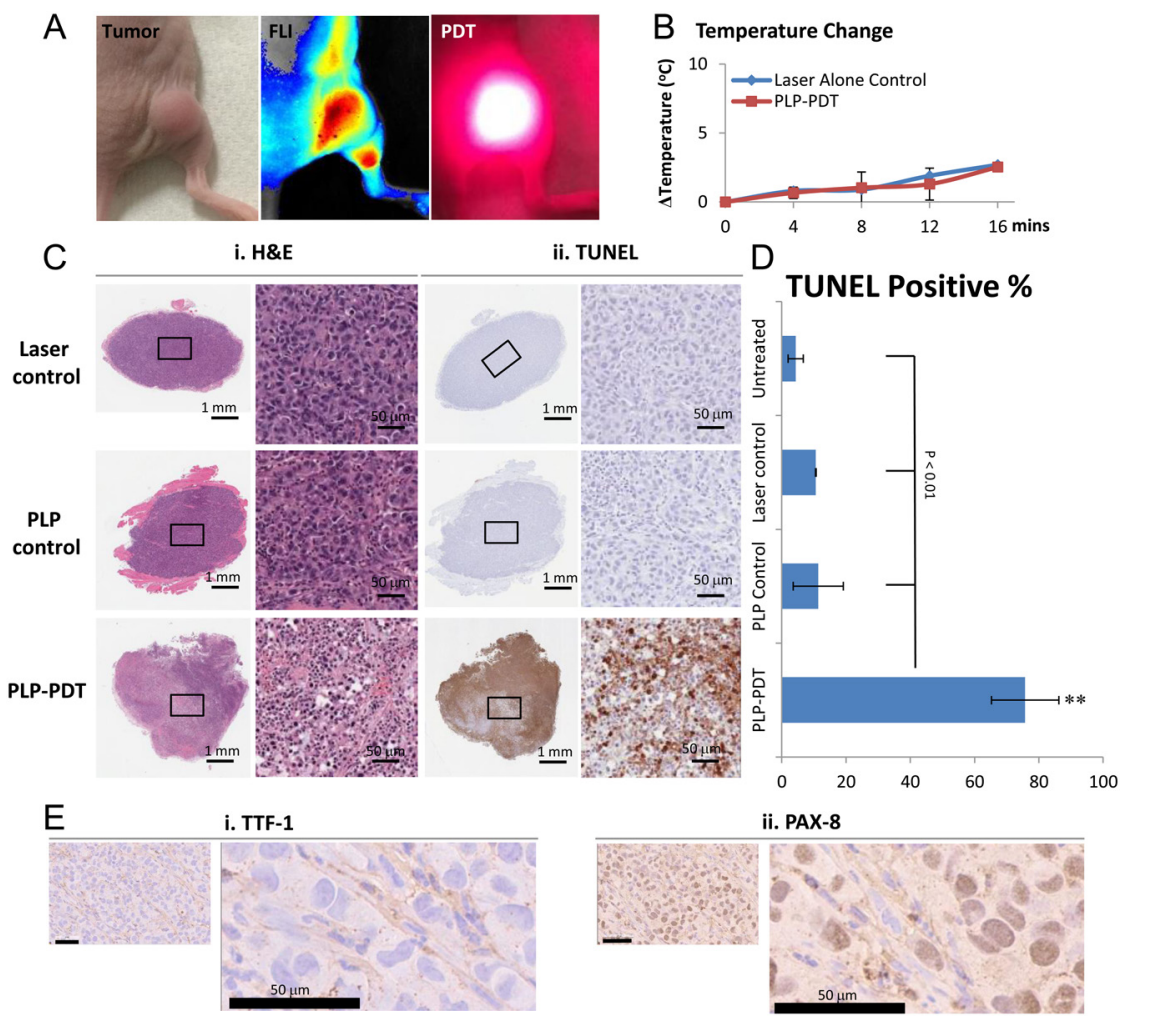

\section{Figure 2}

PLP accumulation and PDT in a subcutaneous thyroid cancer model. (A) In vivo subcutaneous PTC K1 tumor in a mouse as observed under white light, fluorescence imaging (ex $595 \mathrm{~nm}$, em 630-720 nm), and during laser exposure for PDT. (B) Temperature increase as quantified by thermal imaging camera during PDT treatment. (C) Representative histological sections from tumors at $24 \mathrm{~h}$ post treatment, including H\&E and TUNEL images. (D) Percentage of cells that are positive by TUNEL assay in each treatment group $n=12$ ( $n=3$ per group). (E) TTF-1 and PAX-8 histological staining of K1 tumor (Scale $=50 \mu \mathrm{m})$. the temperature of tumors in the PLP-PDT group increased slightly by $2.5 \pm 0.2^{\circ} \mathrm{C}(n=3)$, comparable to that from the laser-alone group (tumor temperature increase by $2.7^{\circ} \mathrm{C}$ $(n=3))$ (Fig. 2B), suggesting no photothermal effect was involved in the treatment as an order of magnitude greater temperature increase is required for photothermal effect (Huang et al. 2006, Jaque et al. 2014). The PDT efficacy to tumor was first evaluated by histological analysis at $24 \mathrm{~h}$ following laser exposure to quantify treatment response at the cellular level (Fig. 2C and D). The TUNEL staining assay revealed that tumor cells from the PLP-PDT group were $75.7 \% \pm 10.5$ positive, while those from laser control, PLP control and untreated control were significantly lower at only $10.6 \% \pm 6.1 \quad(P=0.009), \quad 11.4 \% \pm 7.8 \quad(P=0.004)$ and $4.4 \% \pm 2.4(P=0.009)$ positive, respectively (Fig. $2 \mathrm{D})$. The significantly higher positive rate of TUNEL staining detected in the tumor of PLP-PDT group indicated significant PTC cell apoptosis. K1 tumors were found to be PAX-8 positive and TTF-1 negative, validating their in vivo status as a biologically relevant PTC model as described previously (Fig. 2E) (Schweppe et al. 2008).

Specificity of PLP accumulation in thyroid tumor was further evaluated using ${ }^{64} \mathrm{Cu}$-labeled PLPs on an orthotopic thyroid cancer model. Mice with K1 thyroid tumors (3-7 $\mathrm{mm}$ ) were injected intravenously with ${ }^{64} \mathrm{Cu}$-labeled PLP. Twenty-four hours post injection, animals were killed, organs recovered and PLP concentration (\%ID/g) quantified by gamma counter. As demonstrated in Fig. $3 \mathrm{~A}$ and $\mathrm{B}$, PLP concentration (\%ID/g) was observed to be highest in the liver $(9.66 \pm 1.29 \%)$, with a significant concentration observed in the blood $(4.46 \pm 0.52 \%)$ at $24 \mathrm{~h}$ post injection, similar to described previously for PLP (Cui et al. 2015). However, as apparent in Fig. 3A, in the area of interest near the tumor and thyroid, K1 tumor had significantly higher ${ }^{64} \mathrm{Cu}$-PLP $(3.81 \pm 0.83 \%, P<0.0001)$ uptake than the contralateral thyroid $(0.88 \pm 0.24 \%)$, muscle $(0.46 \pm 0.14 \%$, $P<0.03)$ and trachea $(1.46 \pm 0.51 \%, P<0.0001)$.

\section{Rabbit model results}

\section{PLP-enabled fluorescence imaging in anatomically relevant rabbit thyroid tumor model}

We next evaluated the capability of PLP to delineate tumors based on its intrinsic fluorescence imaging properties in an anatomically relevant rabbit thyroid cancer model (VX2 tumor-implanted in rabbit thyroid gland). To visualize tumor in the thyroid, a small incision was made at the neck area to expose the thyroid and surrounding tissues (Fig. 4Ai). Twenty-four hours following administration of $4 \mathrm{mg} / \mathrm{kg}$ PLP via marginal ear vein injection, a strong fluorescence signal was detected under an in-house fluorescence imaging endoscopy system $(650 \pm 20 \mathrm{~nm}$ excitation, $700 \pm 25 \mathrm{~nm}$ (c) 2020 Society for Endocrinology Published by Bioscientifica Ltd. Printed in Great Britain 

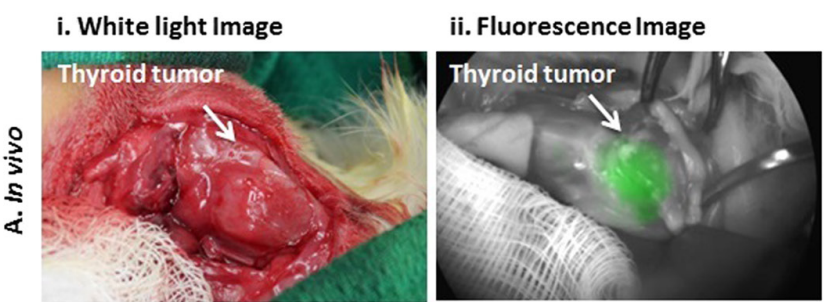

\section{A Orthotopic Thyroid Tumour 24 Hours p.i.}

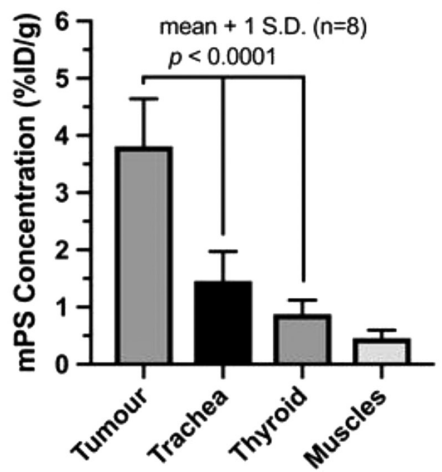

\section{B Major Organ 24 Hours p.i.}

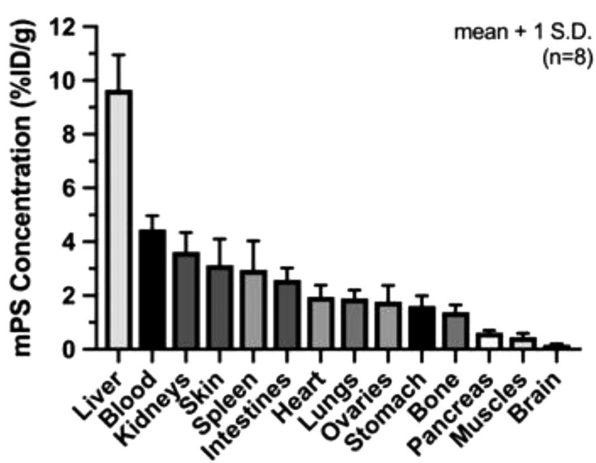

Figure 3

Biodistribution of ${ }^{64} \mathrm{Cu}$-labeled PLP in mouse orthotopic thyroid cancer model at 24 h post injection. (A) PLP concentration (\%lD/g) in target and normal proximal tissues (trachea, thyroid, muscle). (B) Biodistribution of PLP in main organs.

emission) (Fig. 4Aii). Fluorescence was subsequently used to guide resection of tumor and surrounding tissues, followed by ex vivo imaging with a Maestro fluorescence imaging system (Fig. 4Bi). In vivo and ex vivo fluorescence imaging were consistent in demonstrating visibly higher PLP accumulation in tumor tissue relative to the surrounding thyroid gland and muscle (Fig. 4Bi and Bii). The tumor tissues were further subjected to histological analysis, with pancytokeratin and H\&E staining confirming the epithelial origins of the tissue (Fig. 4C, Di and Ei). Additionally, microscopy images of serial section from tumor and surrounding RLN showed clear accumulation of PLP (red) in tumor but not in nerve tissue (Fig. 4Dii and Eii).

\section{PLP-enabled effective PDT in rabbit VX2 thyroid tumor model}

Following confirmation of the selective accumulation of PLP in thyroid tumor, we continued investigating PLP-mediated PDT efficacy using the rabbit VX2 thyroid tumor model. When tumors reached one centimeter in
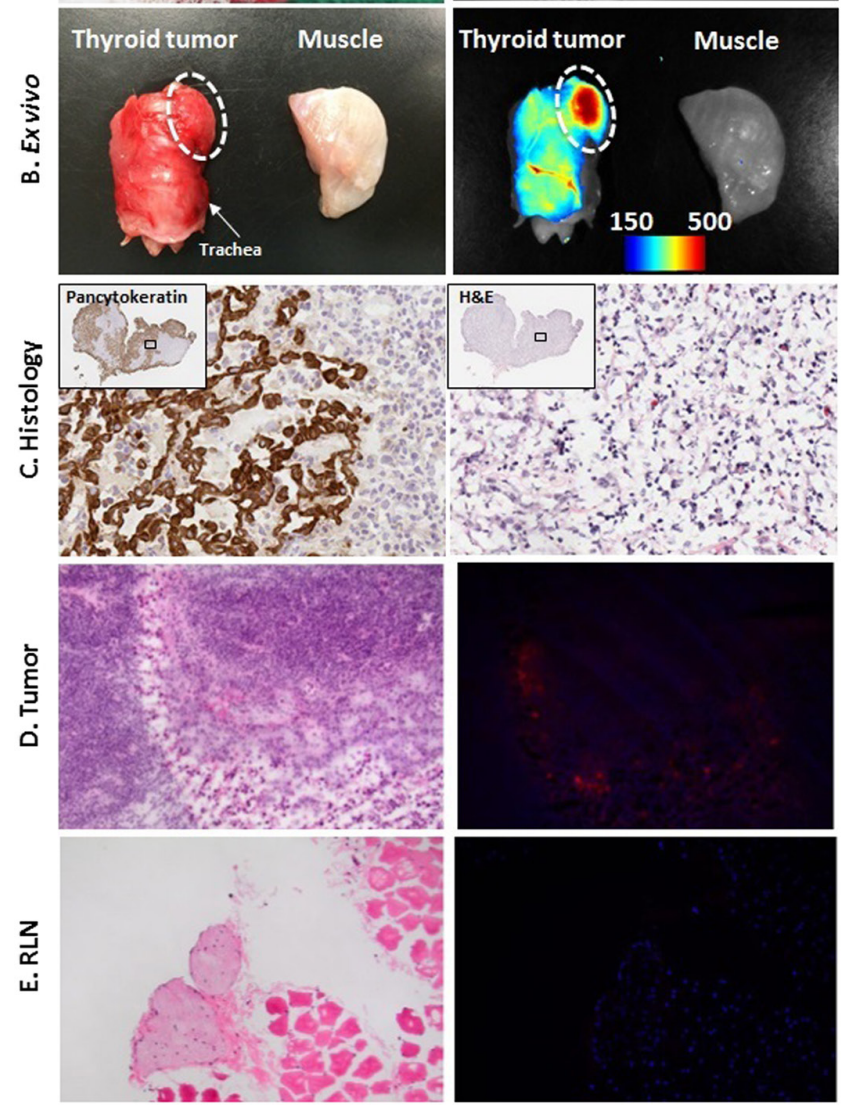

\section{Figure 4}

Specific accumulation of porphysome in rabbit thyroid tumor. (A) In vivo imaging of a VX2 thyroid tumor in rabbit using white light (Ai) and fluorescence imaging (Aii). (B) Ex vivo fluorescence imaging of rabbit thyroid tumor and proximal normal tissues including trachea, larynx and muscle. (C) Pancytokeratin and H\&E staining were used to verify epithelial origin of the tissue. (D and E) H\&E-stained tumor (Di) and RLN (Ei) and corresponding fluorescent microscopy images with PLP appearing red in tumor (Dii) and unobservable in RLN (Eii).

diameter (as measured by CT), $4 \mathrm{mg} / \mathrm{kg}$ PLP was injected intravenously. At $24 \mathrm{~h}$ post injection, tumors were exposed by surgical incision through the overlying skin and strap muscle and received laser irradiation following a laser fiber insertion method depicted schematically in the Fig. 5Ai. Laser irradiation alone and PLP-PDT caused minimal temperature increase in tumor by $2.1 \pm 1.4^{\circ} \mathrm{C}$ and $1.8 \pm 0.6$, respectively, indicating no photothermal effect caused by laser irradiation in either group. Short-term PDT efficacy was evaluated by histological analysis of tumor and tissues 
at $24 \mathrm{~h}$ following irradiation (Fig. 5C, D and E). In contrast to the minimal amount of tumor cell damage that was observed in the laser control group by both H\&E staining and TUNEL assay (8.23\% positive, $n=3$ ) (Fig. 5Ci and Di), PLP-PDT caused significant tumor tissue damage and apoptosis (TUNEL 66.39 $\pm 0.76 \%$ positive, $n=3$ ) (Fig. 5Civ and Div). Additionally, nearby tissues including trachea and RLN appeared undamaged by PDT, evidenced by their normal morphology with H\&E staining (Fig. 5Cii and Ciii) and low levels of apoptosis observed under TUNEL assay (0.83\% TUNEL positive for trachea and negligible TUNEL positive for RLN) (Fig. 5Dii, Diii and E). These data suggest that PLP-PDT is a safe and effective therapeutic approach for thyroid cancer. Moreover, to investigate potential phototoxicity of PLP-PDT to the surrounding RLN nerve bundle, we have exposed RLN directly to laser irradiation and examined the response by histopathological analysis. As shown in Fig. 5B, the left RLN was exposed and received direct laser irradiation (100 $\left.\mathrm{mW}, 100 \mathrm{~J} / \mathrm{cm}^{2}\right)$ at $24 \mathrm{~h}$ after the rabbits received PLP ( $4 \mathrm{mg} / \mathrm{kg}$ ) intravenously. Twentyfour hours post PDT, the RLNs from both sides (rightside: dark control, left-side: light-treated) were collected and subjected to histology analysis. As shown in Fig. 5F and G, both sides of RLNs exhibited normal morphology with H\&E staining and minimal apoptosis under TUNEL staining, indicating the safety of PLP-PDT to normal RLN.

\section{Long-term survival of rabbits with VX2 thyroid tumor after PDT treatment}

The long-term therapeutic efficacy of PLP-PDT was assessed by tumor growth and survival rate of rabbits. Additionally, the RLN function was further investigated by endoscopic assessment of vocal cord movement. When tumors reached an approximate $1 \mathrm{~cm}$ in diameter, VX2 thyroid tumor bearing rabbits were divided into three groups: untreated control $(n=3)$, laser control $(n=3)$ and PLP-PDT group $(n=3)$. As shown in Fig. 6 , the tumors in the untreated control and the laser control groups grew rapidly, triggering tumor size control endpoint $(3 \mathrm{~cm}$ diameter) or causing eating difficulty of the animals that eventually leads to weight loss endpoint (20\% loss of weight) for all animals from the days 14-21 in the control groups (Fig. 6A and B). In contrast, the tumor size in the PLP-PDT group slightly increased within 7 days after treatment, likely due to the edema induced by the PDT apoptosis and necrosis (the dark area detected by CT on the Day 21, Fig. 6Ai), followed by a gradual decrease in size as edema was reduced and cell debris was cleared (Fig. 6C). On the day 53 (the day 39 post-treatment), rabbits treated by PLP-PDT were observed to be disease free as no tumor was detected at the neck area by CT scanning. The tumorfree status of the PLP-PDT group was further confirmed by thyroid tissue histology analysis after killing these animals at the day 53 and resecting their thyroid for evaluation (Fig. 6D). Only normal thyroid gland tissue remained with no tumor cells were detected histologically, confirming that the thyroid tumor was completely ablated by PLPPDT while sparing normal thyroid tissue from damage. In addition, to further investigate local soft tissue toxicity side effects, the functional status of the RLN was assessed by comparing vocal cord movement using endoscopy prior to and post treatment (at $24 \mathrm{~h}$ and a week post treatment). After PLP-PDT treatment, the vocal cords showed normal and symmetrical movement at $24 \mathrm{~h}$ post PDT (Supplementary Fig. 2), further indicating the safety of PLP-PDT to local soft tissues.

\section{Discussion}

The incidence of thyroid cancer is increasing each year. Although the current gold standard for treatment is surgery, there is increasing interest in alternative techniques that can reduce morbidity while maintaining the high survival rates associated with surgical treatment of differentiated thyroid cancer (Davies \& Welch 2014, Haugen et al. 2016). Many recent clinical studies have focused on new surgical techniques to treat thyroid cancer including transaxillary, transoral and translabial approaches (Russell et al. 2019). These novel techniques have been developed so as to avoid a surgical scar on the neck; however, they also result in the traditional morbidities associated with standard thyroid surgery including, hypocalcaemia, hypoparathyroidism, recurrent laryngeal nerve damage, post operative fever, hematoma/hemorrhage, wound infection, thromboembolic events and cardiopulmonary events (Papaleontiou et al. 2017).

With the goal of developing alternative therapeutic methods to minimize morbidity associated with normal thyroid, RLN or parathyroid damage, we have developed an alternative minimal access nanoparticle-based approach to ablate thyroid cancer. We have incorporated multiple endpoints to assess the impact of PLP-PDT on normal thyroid tissue and the RLN. We evaluated the pre-clinical efficacy and safety of a PLP-PDT for treatment of thyroid cancer on both a biologically relevant thyroid tumor mouse model and an anatomically relevant VX2 tumorimplanted rabbit thyroid model. The results demonstrate (c) 2020 Society for Endocrinology Published by Bioscientifica Ltd. Printed in Great Britain 
A In vivo PDT

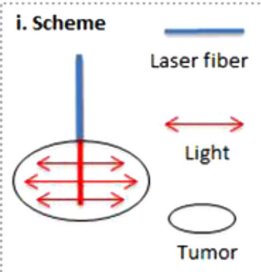

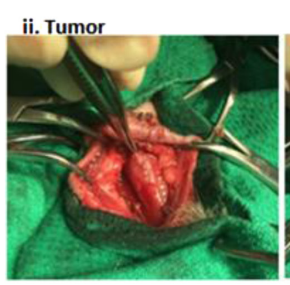

C H\&E
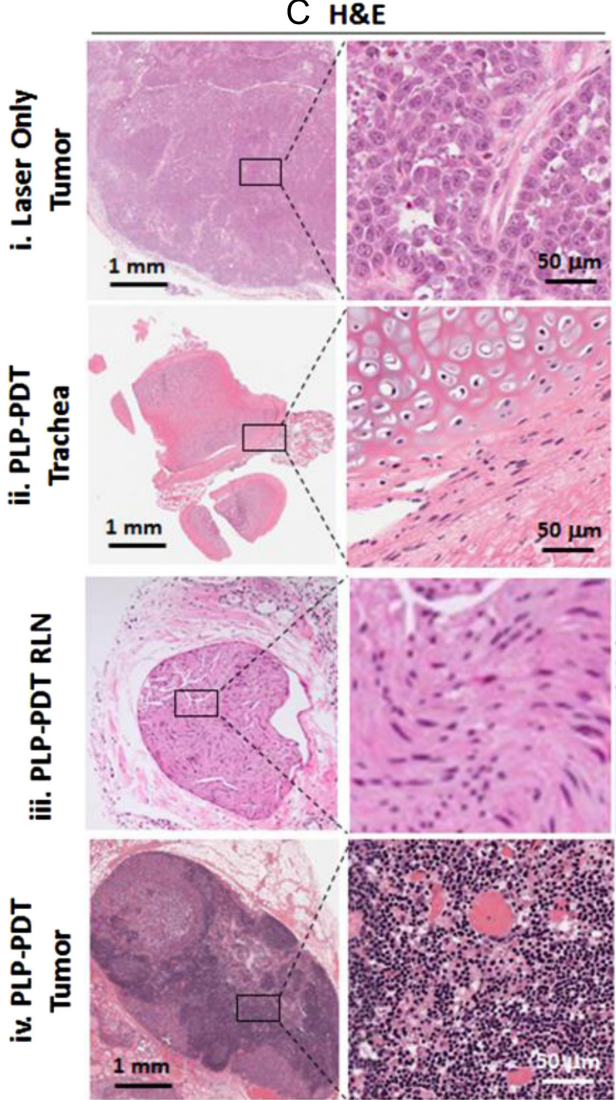

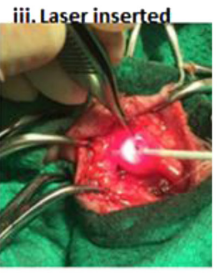

B Direct Laser irradiation to RLN $\left(100 \mathrm{~mW}, 100 \mathrm{~J} / \mathrm{cm}^{2}\right)$
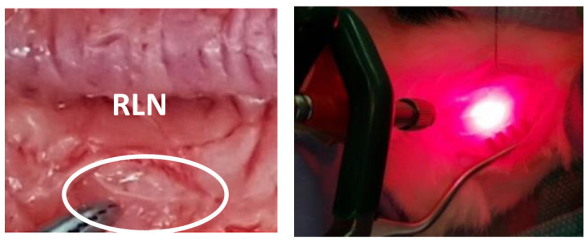

D TUNEL

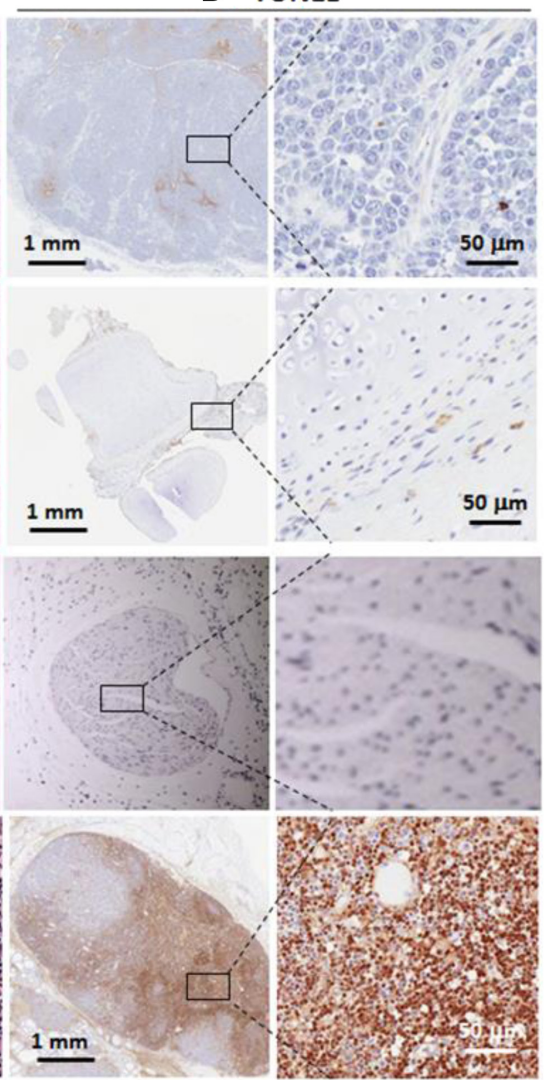

E TUNEL Positive \%

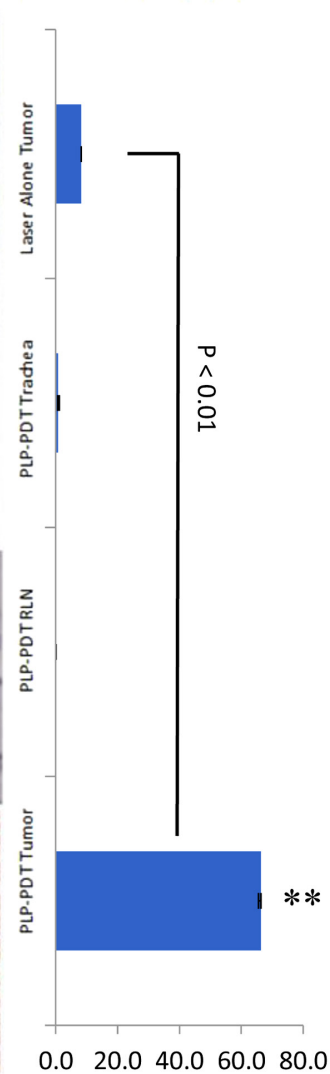

G Left RLN (PDT-treated)
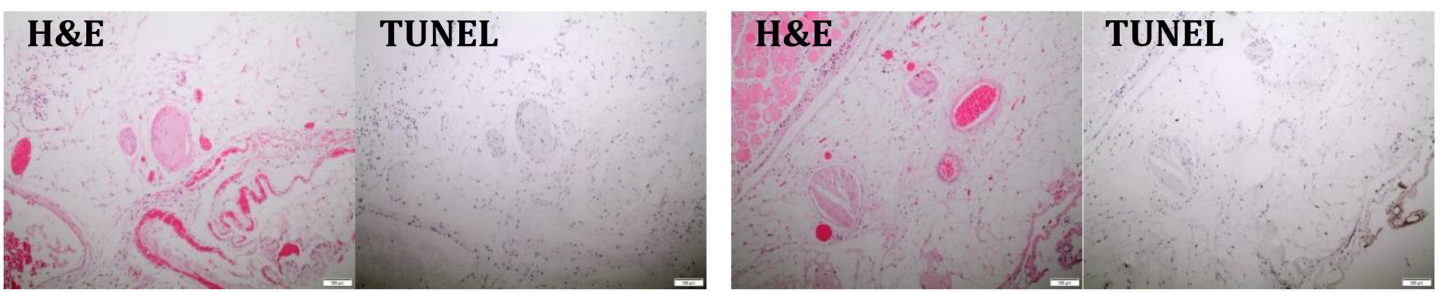

\section{Figure 5}

PLP-PDT treatment in rabbit VX2 thyroid tumor model. (A) Schematic of laser exposure (Ai) and, image of thyroid tumor (Aii) and laser irradiation of thyroid tumor (Aiii). (B) Direct laser irradiation to left-side RLN of health rabbit after $24 \mathrm{~h}$ receiving PLP administration. (C) H\&E images of rabbit thyroid tumor or trachea following PDT. (D) Images from TUNEL assay of tissue from same serial section as H\&E images. (E) Quantification of TUNEL-positive cell population in response to treatment $(n=11, P<0.01)$. ( $F$ and $\mathrm{G})$ H\&E and TUNEL staining images of RLN tissues of healthy rabbits after receiving direct PLP-PDT to left-side of RLN: (F) images of right-side (dark control) RLN; (G) images of left-side (light-treated) RLN. 

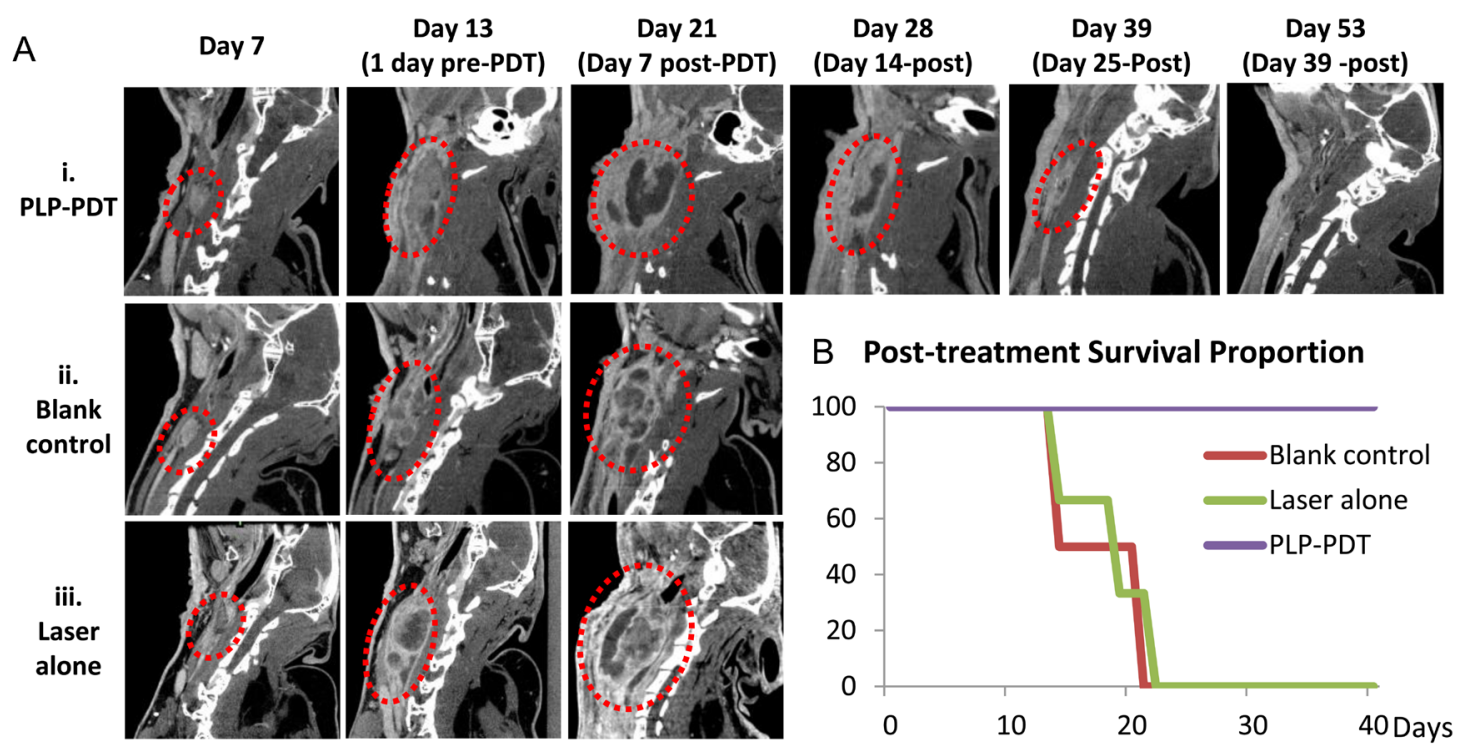

\section{Individual tumor growth curve}
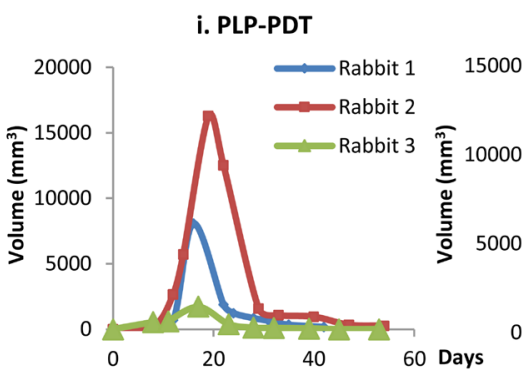

ii. Tumor Alone Control

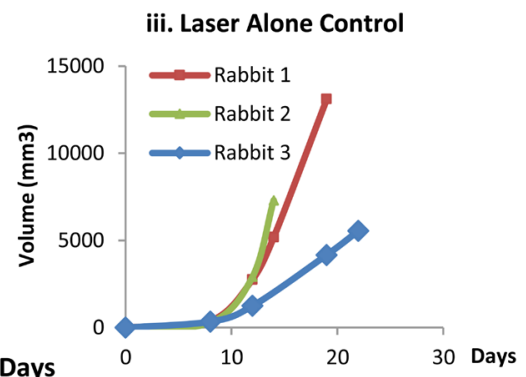

D Histology analysis of thyroid post PLP-PDT

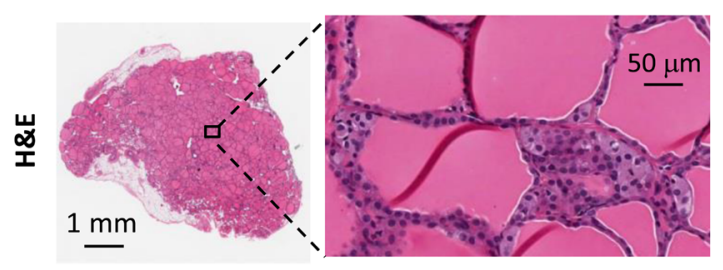

\section{Figure 6}

Longitudinal response of VX2 thyroid tumors to PLP-mediated PDT. (A) CT images of tumor in different groups (PLP-PDT, blank control, laser control). All rabbits in the untreated and laser-alone control groups reached the end-point before or on the Day 21 (the Day 7 post treatment). (B) Post-treatment survival proportion (\%). (C) Tumor growth curves of individual animals in each PDT treatment and control groups. (D) Histological images of remaining thyroid gland of a rabbit that received the PLP-PDT treatment.

a number of advantageous properties including specific passive accumulation of PLP in thyroid tumor tissue and, specific and potent PLP-mediated destruction to tumor tissue following PDT.

Damage to the recurrent laryngeal nerve is an infrequent but significant complication of thyroid cancer surgery and is reported to be as high as $12 \%$ of patients in a large analysis of SEER data, with surgeon volume, patient age and disease stage impacting this rate (Landerholm et al. 2014, Papaleontiou et al. 2017).
As such, any new treatment must ensure that this level of morbidity is reduced. In this study, examination of normal thyroid, trachea and RLN tissue following PDT to the tumor did not reveal any histological damage by H\&E and TUNEL assay, implying that the PDT did not cause any structural damage of these three normal tissue types. We further assessed the potential phototoxicity to the RLN nerve by exposing the nerve directly to laser irradiation after injection of the PLP. Twenty-four hours post laser exposure, the RLNs from both sides were subjected to (c) 2020 Society for Endocrinology Published by Bioscientifica Ltd. Printed in Great Britain 
histological analysis demonstrating normal morphology with H\&E staining and minimal apoptosis with TUNEL staining, further emphasizing the safety of PLP-PDT to the normal RLN.

The American Thyroid Association (ATA) guidelines recommend assessment of vocal cord function with flexible endoscopy pre and post surgery. We assessed rabbit RLN function via direct endoscopy to evaluate vocal cord movement following PDT treatment (Smith et al. 2014). It is recognized that the pre-clinical model used here did not allow for a full clinical assessment of vocal cord function. However, such assessment consisting of electromyography and stroboscopy during phonation is rarely completed pre and post thyroid surgery in clinical practice either. Therefore, we performed the current clinical standard of vocal cord assessment, as per the ATA guidelines, on the rabbit pre clinical model. There was no change in the rabbit vocal cord movement post treatment, when compared to their pre treatment assessment. The combination of lack of nanoparticle accumulation and histological damage to any normal tissue examined, and the observation of normal vocal cord movement postPDT, indicate that cellular toxicity was limited to thyroid tumor tissue.

Although radiofrequency, laser and alcohol ablation techniques have been used for decades, and more recently included as alternative treatment modalities for a subset of thyroid cancer patients in the 2015 American Thyroid Association (ATA) guidelines, they are still not commonly utilized in most cancer treatment centers (Haugen et al. 2016, Nixon et al. 2018). Indeed, the 2015 ATA guidelines acknowledge that radiofrequency, alcohol and laser ablation should primarily be considered as alternatives only in high-risk patients with recurrence or patients refusing surgery, not as a standard alternative to surgical resection. This is in large part due to the requirement for multiple treatments and complications including pain, skin burns and voice changes (Haugen et al. 2016, Nixon et al. 2018). Furthermore, such treatments are not tumor specific. In comparison, a potentially significant advantage of PLP-PDT treatment is the high level of cytotoxic specificity gained from the combination of preferential particle accumulation in tumor, controlled location of laser exposure, reduced laser power and limited spatiotemporal range of cytotoxicity due to the mechanism of PDT relying on generation of reactive oxygen species (ROS) instead of temperature change or dehydration for cytotoxicity. PDT controls the localization of tissue damage only to cancer cells that receive the combination of sufficient PLP nanoparticle accumulation and light of the appropriate wavelength to generate ROS with the radius of action for singlet oxygen reported to be 10-20 nm (Moan \& Berg 1991). This restriction of cytotoxicity to tumor tissue that contains PLP appears to have been achieved as we observed complete ablation of tumors in rabbit thyroid while maintaining normal thyroid, trachea and RLN histology and function.

Cosmesis of the visible surgical scar on the neck has been shown to negatively impact on a patient's quality of life (Choi et al. 2014, Arora et al. 2016). As such, it should be noted that while the PDT procedure described here was performed primarily to determine specificity and efficacy of tumor ablation without focusing on the invasiveness of the procedure, the PLP-PDT procedure can be performed with minimal invasiveness compared to standard surgery. It is anticipated that PLP-PDT can be performed using ultrasound-guided catheter insertion of the laser fiber directly into the tumor; a technique similar to fine-needle aspiration biopsy (FNAB) of a thyroid nodule. This would allow focused treatment of the thyroid cancer while ensuring that there was no surgical scar on the neck and no significant tissue manipulation as seen in surgery. Additionally, as demonstrated in the results, normal thyroid and the RLN are expected to be undamaged due to minimal accumulation of nanoparticle in these tissues and no observable histological damage when exposed directly to a $671 \mathrm{~nm}$ PDT laser (Figs 4 and 5).

A limitation of this study is that we did not assess the histology or function of the parathyroid glands in either animal model following PDT. In humans, a surrogate marker of parathyroid gland function is adjusted calcium levels. However, in rabbits, calcium metabolism is different from humans and the levels measured only reflect the levels of calcium they are taking in their diet; therefore, this marker is not suitable for assessment of parathyroid function in rabbits (Redrobe 2002).

Overall, the results presented here demonstrate several advantageous properties of PLP-PDT for treatment of thyroid cancer, including being a theranostic method that resulted in complete tumor ablation, no damage to the recurrent laryngeal nerve and preservation to normal thyroid tissue. Furthermore, this porphyrin nanoparticlemediated PDT treatment method has the potential to be delivered via a minimally invasive, ultrasound-guided approach using local anesthetic and laser fiber placement in a very similar technique to FNAB. The results presented here support continued research and development of this PLP-PDT method as an alternative, beneficial and highly specific method of treating thyroid cancer. 


\section{Supplementary materials}

This is linked to the online version of the paper at https://doi.org/10.1530/ ERC-19-0258.

\section{Declaration of interest}

The authors declare that there is no conflict of interest that could be perceived as prejudicing the impartiality of the research reported.

\section{Funding}

This work is supported by the Terry Fox Research Institute (PPG\#1075), the Princess Margaret Cancer Foundation - Epstein GTx Research Partnership Fund, the Canadian Cancer Society Research Institute (704718), the Canadian Institute of Health Research (Foundation Grant \#154326), and the Canada Foundation for Innovation, and the Princess Margaret Cancer Foundation.

\section{Acknowledgements}

The authors would like to acknowledge the Spatio-Temporal Targeting and Amplification of Radiation Response (STTARR) program and its affiliated funding agencies.

\section{References}

Arora A, Swords C, Garas G, Chaidas K, Prichard A, Budge J, Davies DC \& Tolley N 2016 The perception of scar cosmesis following thyroid and parathyroid surgery: a prospective cohort study. International Journal of Surgery 25 38-43. (https://doi.org/10.1016/j. ijsu.2015.11.021)

Betz CS, Jager HR, Brookes JA, Richards R, Leunig A \& Hopper C 2007 Interstitial photodynamic therapy for a symptom-targeted treatment of complex vascular malformations in the head and neck region. Lasers in Surgery and Medicine 39 571-582. (https://doi.org/10.1002/ $1 \mathrm{sm} .20535)$

Choi Y, Lee JH, Kim YH, Lee YS, Chang HS, Park CS \& Roh MR 2014 Impact of postthyroidectomy scar on the quality of life of thyroid cancer patients. Annals of Dermatology 26 693-699. (https://doi. org/10.5021/ad.2014.26.6.693)

Cui L, Lin Q, Jin CS, Jiang W, Huang H, Ding L, Muhanna N, Irish JC, Wang F, Chen J, et al. 2015 A pegylation-free biomimetic porphyrin nanoplatform for personalized cancer theranostics. ACS Nano 9 4484-4495. (https://doi.org/10.1021/acsnano.5b01077)

Davies L \& Welch HG 2014 Current thyroid cancer trends in the United States. JAMA Otolaryngology: Head and Neck Surgery 140 317-322. (https://doi.org/10.1001/jamaoto.2014.1)

Gambelunghe G, Fede R, Bini V, Monacelli M, Avenia N, D'ajello M, Colella R, Nasini G \& De Feo P 2013 Ultrasound-guided interstitial laser ablation for thyroid nodules is effective only at high total amounts of energy: results from a three-year pilot study. Surgical Innovation 20 345-350. (https://doi.org/10.1177/1553350612459276)

Haugen BR, Alexander EK, Bible KC, Doherty GM, Mandel SJ, Nikiforov YE, Pacini F, Randolph GW, Sawka AM, Schlumberger M, et al. 20162015 American Thyroid Association management guidelines for adult patients with thyroid nodules and differentiated thyroid cancer: the American Thyroid Association Guidelines Task Force on thyroid nodules and differentiated thyroid cancer. Thyroid 26 1-133. (https://doi.org/10.1089/thy.2015.0020)
Huang X, Jain PK, El-Sayed IH \& El-Sayed MA 2006 Determination of the minimum temperature required for selective photothermal destruction of cancer cells with the use of immunotargeted gold nanoparticles. Photochemistry and Photobiology 82 412-417. (https:// doi.org/10.1562/2005-12-14-RA-754)

Ito Y, Kudo T, Kobayashi K, Miya A, Ichihara K \& Miyauchi A 2012 Prognostic factors for recurrence of papillary thyroid carcinoma in the lymph nodes, lung, and bone: analysis of 5,768 patients with average 10-year follow-up. World Journal of Surgery 36 1274-1278. (https://doi.org/10.1007/s00268-012-1423-5)

Jaque D, Martinez Maestro L, Del Rosal B, Haro-Gonzalez P, Benayas A, Plaza JL, Martin Rodriguez E \& Garcia Sole J 2014 Nanoparticles for photothermal therapies. Nanoscale 6 9494-9530. (https://doi. org/10.1039/c4nr00708e)

La Vecchia C, Malvezzi M, Bosetti C, Garavello W, Bertuccio P, Levi F \& Negri E 2015 Thyroid cancer mortality and incidence: a global overview. International Journal of Cancer 136 2187-2195. (https://doi. org/10.1002/ijc.29251)

Landerholm K, Wasner AM \& Jarhult J 2014 Incidence and risk factors for injuries to the recurrent laryngeal nerve during neck surgery in the moderate-volume setting. Langenbeck's Archives of Surgery 399 509-515. (https://doi.org/10.1007/s00423-013-1154-6)

Lim H, Devesa SS, Sosa JA, Check D \& Kitahara CM 2017 Trends in thyroid cancer incidence and mortality in the United States, 19742013. JAMA 317 1338-1348. (https://doi.org/10.1001/ jama.2017.2719)

Lin LM, Chen YK, Chen CH, Chen YW, Huang AH \& Wang WC 2009 VX2-induced rabbit buccal carcinoma: a potential cancer model for human buccal mucosa squamous cell carcinoma. Oral Oncology 45 e196-e203. (https://doi.org/10.1016/j.oraloncology.2009.07.012)

Moan J \& Berg K 1991 The photodegradation of porphyrins in cells can be used to estimate the lifetime of singlet oxygen. Photochemistry and Photobiology 53 549-553. (https://doi.org/10.1111/j.1751-1097.1991. tb03669.x)

Muhanna N, Cui L, Chan H, Burgess L, Jin CS, Macdonald TD, Huynh E, Wang F, Chen J, Irish JC, et al. 2015a Multimodal image-guided surgical and photodynamic interventions in head-and-neck cancer: from primary tumor to metastatic drainage. Clinical Cancer Research 22 961-970. (https://doi.org/10.1158/1078-0432.CCR-15-1235)

Muhanna N, Jin CS, Huynh E, Chan H, Qiu Y, Jiang W, Cui L, Burgess L, Akens MK, Chen J, et al. 2015b Phototheranostic porphyrin nanoparticles enable visualization and targeted treatment of head and neck cancer in clinically relevant models. Theranostics $\mathbf{5}$ 1428-1443. (https://doi.org/10.7150/thno.13451)

Nixon IJ, Angelos P, Shaha AR, Rinaldo A, Williams MD \& Ferlito A 2018 Image-guided chemical and thermal ablations for thyroid disease: review of efficacy and complications. Head and Neck $\mathbf{4 0}$ 2103-2115. (https://doi.org/10.1002/hed.25181)

Pacella CM, Mauri G, Achille G, Barbaro D, Bizzarri G, De Feo P, Di Stasio E, Esposito R, Gambelunghe G, Misischi I, et al. 2015 Outcomes and risk factors for complications of laser ablation for thyroid nodules: a multicenter study on 1531 patients. Journal of Clinical Endocrinology and Metabolism 100 3903-3910. (https://doi. org/10.1210/jc.2015-1964)

Papaleontiou M, Hughes DT, Guo C, Banerjee M \& Haymart MR 2017 Population-based assessment of complications following surgery for thyroid cancer. Journal of Clinical Endocrinology and Metabolism 102 2543-2551. (https://doi.org/10.1210/jc.2017-00255)

Parvinian A, Casadaban LC \& Gaba RC 2014 Development, growth, propagation, and angiographic utilization of the rabbit VX2 model of liver cancer: a pictorial primer and 'how to' guide. Diagnostic and Interventional Radiology 20 335-340. (https://doi.org/10.5152/ dir.2014.13415)

Redrobe S 2002 Calcium metabolism in rabbits. Seminars in Avian and Exotic Pet Medicine 11 94-101. (https://doi.org/10.1053/ saep.2002.125100) 
Rous P, Kidd JG \& Smith WE 1952 Experiments on the cause of the rabbit carcinomas derived from virus-induced papillomas. II. Loss by the Vx2 carcinoma of the power to immunize hosts against the papilloma virus. Journal of Experimental Medicine 96 159-174. (https://doi.org/10.1084/jem.96.2.159)

Russell JO, Razavi CR, Garstka ME, Chen LW, Vasiliou E, Kang SW, Tufano RP \& Kandil E 2019 Remote-access thyroidectomy: a multiinstitutional North American experience with transaxillary, robotic facelift, and transoral endoscopic vestibular approaches. Journal of the American College of Surgeons 228 516-522. (https://doi. org/10.1016/j.jamcollsurg.2018.12.005)
Schweppe RE, Klopper JP, Korch C, Pugazhenthi U, Benezra M, Knauf JA, Fagin JA, Marlow LA, Copland JA, Smallridge RC, et al. 2008

Deoxyribonucleic acid profiling analysis of 40 human thyroid cancer cell lines reveals cross-contamination resulting in cell line redundancy and misidentification. Journal of Clinical Endocrinology and Metabolism 93 4331-4341. (https://doi.org/10.1210/jc.20081102)

Smith J, Douglas J, Smith B, Dougherty T \& Ayshford C 2014 Assessment of recurrent laryngeal nerve function during thyroid surgery. Annals of the Royal College of Surgeons of England 96 130-135. (https://doi.org/10.1308/003588414X13814021676594)

Received in final form 13 November 2019

Accepted 18 November 2019

Accepted Manuscript published online 18 November 2019
C) 2020 Society for Endocrinology Published by Bioscientifica Ltd. Printed in Great Britain 